\title{
Heuristics and Heterogeneity in Health Insurance Exchanges: Evidence from the Massachusetts Connector
}

This Draft: January 1, 2012

By Keith Marzilli ERicson And Amanda StarC*

* Ericson: Boston University, 595 Commonwealth Ave., Boston, MA 02215 (e-mail: kericson@bu.edu). Starc: The Wharton School, University of Penn., 3641 Locust Walk Philadelphia, PA 19104 (email: astarc@wharton.upenn.edu). We thank David Cutler, Jon Gruber, and Jim Rebitzer for helpful discussions.

Although health insurance exchanges are a growing source of individual coverage, little is known about consumer behavior on these exchanges. While health insurance exchanges are intended to facilitate competition among insurers and make it easier for consumers to access insurance, understanding how consumers choose insurance plans is necessary for the design of these markets. We characterize demand on a health insurance exchange, including the type of plans individuals choose and how they respond to price. Previous work has noted the potential limits to consumer decision making ability in similar environments (Abaluck and Gruber 2011). In a structural model of health plan choice, we consider the role of heterogeneity in preferences and whether consumers rely on simple rules of thumb.
We offer a first look at behavior on Massachusetts' unsubsidized health insurance exchange, the "Connector", 1 which was created as a result of the 2006 Massachusetts health reform. A forerunner for the 2010 federal health reform, the Massachusetts reform established a state-wide health insurance exchange and an individual mandate to obtain insurance. The exchange offers health insurance to individuals who were not offered insurance by their employer and who do not receive state-subsidized insurance, and accounts for approximately $1 \%$ of the $5 \%$ reduction in the uninsured following reform (Kolstad and Kowalski 2010).

Our data comprises the complete set of choices made by individuals who purchase unsubsidized insurance through the Connector from its start in 2007 through Dec. 2009. ${ }^{2}$ We

\footnotetext{
1 The full name is the Massachusetts Commonwealth Health Insurance Connector Authority, which run both the unsubsidized "Commonwealth Choice" market considered here, as well as a separate market called "Commonwealth Care" for people whose family income is below $300 \%$ of the federal poverty line.

2 The Connector began offering insurance May 2007, and the mandate took effect July 1, 2007.
} 
supplement this data with detailed price quotes taken in Nov. and Dec. 2009, which allow us to capture the set of prices enrollees face. Consumers pick a plan from the set of plans available to them at posted prices, which vary by age, zip code and family size. They keep the plan they choose at the same monthly premium for a year, unless they leave the Connector; they cannot switch plans within the Connector. Our data is described in further detail in Ericson and Starc (2011).

Insurers offer plans through the exchange, and a given firm will offer plans of varying generosities. The Connector groups these plans into tiers by actuarial value, and consumers see them categorized as Bronze (least generous), Silver, or Gold (most generous). ${ }^{3}$ The actuarial value (percent of health costs insured for a representative individual) of bronze plans is about 55\%; the actuarial value for silver plans can range from $70-80 \%$, and gold from 85 to $95 \% .{ }^{4}$

Table 1 summarizes the choices enrollees make in the Connector, for both the full sample and our Nov.-Dec. 2009 Analysis

3 Additionally, there is a separate set of Young Adult Plans (YAPs), which are less generous than the bronze plans and available only to those aged 26 and under. YAP s account for approximately 30 percent of enrollment in the Connector.

${ }^{4}$ See Peterson (2009). Also see, "Commonwealth Choice Seal of Approval Recommendations,” Massachusetts Connector Authority Board of Directors Meeting. Slide \#26. June 23, 2009.
Sample. ${ }^{5}$ A majority of enrollees (63\%) choose a bronze tier plan, the lowest level of generosity sufficient to satisfy the mandate ("minimum credible coverage"). Since the minimum credible coverage regulation determines the type of plan the majority of consumers hold, it will be a crucial policy decision.

The plans chosen by consumers in the exchange are distinct from the typical employer-sponsored insurance (ESI) plan. The bronze plan chosen by a majority of exchange enrollees is less generous than the the average ESI plan. While Bronze plans have an actuarial value of about 55\%, typical employer-sponsored HMO and PPO plans have actuarial values of $80-84 \%$ and $93 \%$, respectively (Peterson 2009). The types of plans chosen in ESI environments are therefore unlikely to be representative of the plans individuals choose on health insurance exchanges.

The differential tax treatment of insurance accounts for only part of this difference. While ESI is paid for with pre-tax dollars, exchange policies are bought with post-tax dollars. Thus, the marginal cost of buying a more generous ESI plan is subsidized at an

\footnotetext{
5 Our Analysis Sample is limited to individuals 27 and older, who enroll in the Connector for the first time during the months in which we have detailed price data, and who choose individual price coverage.
} 
individual's marginal tax rate; there is no such subsidy on the exchange. However, when we use our estimated models to simulate the plans that would be chosen if prices were reduced to $70 \%$ of current levels, the fraction choosing bronze plans only falls slightly: from 63\% down to $44 \%$ to $60 \%$, depending on specification. ${ }^{6}$ Note this analysis is only a rough approximation of the pricing difference between ESI and the exchange: the exchange has age-based pricing (while ESI does not), firm prices may respond to the subsidy, and employers may offer additional subsidies for choosing more generous insurance.

Nonetheless, the difference in generosity of insurance chosen speaks against a theory of uninsurance in which the uninsured have similar demand to the insured, but are prevented from getting coverage by market frictions (see also Krueger and Kuziemko 2011). Enrollees may choose different plans on the exchange because their underlying preferences differ from ESI recipients. However, purchases on the exchange may differ for other reasons: for instance, since the median enrollee tenure in the Connector is about one year, individuals may be looking for “stop-gap” coverage while they wait for a preferable coverage source.

\footnotetext{
6 Even if prices were reduced to $40 \%$ of current levels, the fraction of individuals choosing bronze is predicted to be $22 \%$ to $57 \%$, depending on specification.
}

A range of evidence suggests that people use heuristics to make difficult decisions (see Gigerenzer and Gaissmaier 2010 for a review). We therefore generalize a structural model of health plan choose to allow individuals to rely on heuristics. Motivated by the fact that approximately $20 \%$ of enrollees choose the cheapest plan available to them, ${ }^{7}$ we focus on one particular heuristic: "choose the cheapest plan.” There are two factors that make this a plausible heuristic. First, price is simple to evaluate, while other characteristics such as deductible and coinsurance are harder to evaluate and tradeoff against each other. Second, the lowest priced plan is listed first in the list of plans, and may benefit from an increase in salience. ${ }^{8}$ Our baseline estimates indicate that a plan moving from second cheapest to cheapest plan ("the minimum effect”) is equivalent to a \$165 dollar per month reduction in its price.

However, enrollees' choices can also be rationalized by allowing consumers to vary in their price sensitivity. Figure 1 motivates our examination of heterogeneity in price sensitivity by age: the fraction of enrollees choosing bronze plans remains roughly constant throughout the age distribution, while

\footnotetext{
7 Because insurers set prices separately in different areas of the state, the identity of the cheapest plan will vary across zip codes.

8 "Choose the cheapest plan" is also consistent with consumers minimizing the cost of compliance with the mandate.
} 
the marginal cost of choosing a more generous plan nearly doubles. Our estimated model indicates substantial heterogeneity in price sensitivity by age, with the youngest individuals approximately twice as price sensitive as the oldest. We also allow for heterogeneity on unobservables, and estimate substantial variation in price sensitivity. Allowing for these types of heterogeneity, in some specifications the minimum effect becomes statistically insignificant or even negative.

The observed choices are thus consistent with heterogeneity in price sensitivity. However, some of our estimated heterogeneity may in fact arise from the use of heuristics. Our identification essentially results from functional form, as an individual using the heuristic "choose the cheapest plan" is observationally identical to an individual being infinitely price sensitive. (The welfare implications differ, however.) In the absence of dominated choices or violations of the weak axiom of revealed preference, it is a challenge to distinguish heuristics or even random choice from utility maximizing models of behavior (Becker 1962). Thus, while we do not find strong evidence for heuristics here, we cannot rule out a role for them. In contrast, we do find strong evidence of consumer heterogeneity in preferences. Failing to account for this heterogeneity will give misleading results.

A limitation of this paper is potential endogeneity of prices, so our results do not well-identify the absolute level of price sensitivity. Our companion paper, Ericson and Starc (2011), uses a regression discontinuity design to identify response to price and finds higher levels of price sensitivity than we find here. That paper also discusses the market design of exchanges and considers the effects of alternative pricing regulations.

\section{Structural Model of Choice with Heuristics}

Consumers face a discrete choice from a set of insurance plans. This choice may be affected by both the utility consumers receive from plans, as well as the decision process leading to the choice of plan. Our model of consumer demand for insurance plans adapts the conditional logit model to include heuristics. In our model, a consumer $i$ chooses the plan $j$ with the highest decision index $v_{i j}$ from the set of plans available to them. This decision index is the sum of the individual's utility of the plan $u_{i j}$, plus an additive "heuristic effect" $h_{j}: v_{i j}=u_{i j}+h_{i j}$.

Because the heuristic effect is additive, the econometrics are not affected by whether a 
researcher believes a characteristic should be included in $u_{i j}$ or $h_{i j}$. However, the distinction between the utility function and heuristic terms is relevant for welfare analysis, and this framework allows us to consider characteristics that consumers are unlikely to directly value (e.g. a plan's position in the choice set).

We assume that consumer $i$ 's utility of plan $j$ is given by: $u_{i j}=X_{j}^{\prime} \beta-\alpha_{i} p_{i j}+\varepsilon_{i j}$, where $\alpha_{i}$ is the disutility of premiums $p_{i j}$ for individual $i, X_{j}$ is a vector of plan characteristics (such as tier, insurer, or plan fixed effects), and $\varepsilon_{i j}$ is an error term that is i.i.d. extreme value. In our baseline specifications, we estimate a common $\alpha$ for all individuals. We then allow $\alpha$ to vary based on observed characteristics (age) and unobserved characteristics (a random coefficients model).

We examine the possibility the consumers may use the heuristic "choose the cheapest plan” by simply including a zero-one indicator variable that takes on a one if the plan is the cheapest in the consumer's choice set. Dividing the coefficient on this dummy by the coefficient on premiums $(\alpha)$ allows us to quantify the heuristic's effect in dollar terms.

Given the assumptions of the logit demand model, the probability person $i$ purchases plan $j$ is given by

$$
s_{i j}=\frac{\exp \left(v_{i j}\right)}{1+\exp \left(v_{i j}\right)}
$$

which also gives plan enrollment shares $s_{j}$. Given the observed enrollment decisions, the components of the decision index $v_{i j}$ can be estimated via maximum likelihood.

\section{Empirical Results}

\section{[ Insert Table 2 Here]}

Table 2 estimates our model, accounting for an effect of price and a separate effect of being the cheapest plan in an enrollee's option set (a binary indicator for being the minimumpriced plan). Column 1 controls for tier and insurer fixed effects. Column 2 controls for plan-level fixed effects and identifies off of geographic variation in price and the identity of the cheapest plan. Dividing the coefficient on the indicator for the minimum priced plan by $\alpha$ quantifies the effect of being the cheapest plan. This effect is equivalent to an additional price reduction of $\$ 33$ (column 1) or $\$ 165$ (column 2) per month.

Column 3 allows for heterogeneity in price sensitivity by allowing the price coefficient to vary by age. We impose a linear age trend in the price coefficient, though similar results are found when $\alpha$ is allowed to vary by age categories. We find substantial variation in price sensitivity, with the youngest individual 
(age 27) being nearly twice as sensitive to price as a 64 year old: $\alpha_{i}=-1.67$ and -0.95 , respectively. The minimum effect is still significant, in equivalent to a price reduction of \$26/month for a 27 year-old and \$46/month for a 64 year-old.

We allow for further heterogeneity in column 4 by letting the preference for tier vary by age. In column 5 by letting preference for plan vary by age, estimating 25 separate age trends. In both cases, substantial heterogeneity in price sensitivity remains. However, in column 5, the minimum effect becomes insignificant and noisily measured.

\section{[ Insert Table 3 Here]}

Table 3 also allows for heterogeneity in price sensitivity on unobservables: it estimates a random coefficients logit model (a.k.a. mixed logit), in which the premium coefficient takes on a distribution. We impose that coefficient follows a lognormal distribution, so consumers always dislike higher prices.

The results indicate substantial variation in price sensitivity even on unobservables, although our identification largely depends on the functional form we imposed. The estimated effect of being the minimum plan is now negative, though not statistically significantly different from zero. We have explored allowing the minimum effect (as well as the price coefficient) to both take on a distribution; in these cases the mean minimum effect is typically small and not statistically significant from zero. However, our ability to separately identify a distribution of both the price coefficient and the minimum effect is limited.

There results demonstrate substantial evidence for price sensitivity. However, the evidence for the use of heuristics is weaker.

\section{Conclusions}

We find that insurance plans consumers choose in an exchange setting are markedly different than demand in existing markets. Therefore, we argue that studying consumer demand, and, by extension, insurer incentives, in this setting is crucially important. This paper focuses on the fact that consumers gravitate toward the cheapest and least generous plans in the Connector. These results indicate that minimal credible coverage laws will be important in determining the level of coverage individuals receive on the exchange.

In addition, plans that are the cheapest in their area receive a substantial increase in enrollment. We argue that either a heuristic, such as "choose the cheapest plan" or consumer heterogeneity in preferences could rationalize the patterns we see in the data. Consumer heterogeneity is a robust 
phenomenon in this data, and ignoring it will likely lead to misleading results. Ideally, however, models should be estimated that are flexible enough to incorporate both heterogeneity and decision rules that do not depend directly on preferences alone, such as heuristics.

\section{REFERENCES}

Abaluck, J. and Gruber, Jon. 2011. "Choice inconsistencies among the elderly: Evidence from plan choice in the Medicare Part D program.” American Economic Review, 2011, 101, 1180-1210

Becker, Gary. 1962. “Irrational behavior and economic theory". Journal of Political Economy, 70, 1-13.

Ericson, Keith. 2011. "Market Design When Firms Interact with Inertia Consumers: Evidence from Medicare Part D”. Working Paper.

Ericson, K. and A. Starc. 2011. "Consumer Behavior on Health Insurance Exchanges: Age-Based Heterogeneity on the Massachusetts Connector”. Working Paper.

Gigerenzer, G. \& Gaissmaier, W. 2010. "Heuristic Decision Making” Annual Review of Psychology, 62, 451-482. Handel, Benjamin. 2011. “Adverse Selection and Switching Costs in Health Insurance Markets: When Nudging Hurts." Working Paper.

Krueger, A. and I. Kuziemko. 2011. "The Demand for Health Insurance Among Uninsured Americans: Results of a Survey Experiment and Implications for Policy.” NBER Working Paper 16978.

Kolstad, J. and A. Kowalski. 2010. "The impact of an individual health insurance mandate on hospital and preventive care: Evidence from Massachusetts.” NBER Working Paper 16012.

Peterson, Chris. 2009. "Setting and Valuing Health Insurance Benefits”. Congressional Research Service. April 6, 2009. Report R4049. 
TABLE 1-MASS. CONNECTOR DESCRIPTIVE STATISTICS, NOV.-DEC. 2009

\begin{tabular}{lcc}
\hline \hline & Full Sample & Analysis Sample \\
\hline Tier of Plan Chosen (if not Choosing YAP) & & \\
...Bronze & 0.605 & 0.634 \\
...Silver & 0.300 & 0.284 \\
...Gold & 0.095 & 0.082 \\
N & 24,196 & 1059 \\
\hline
\end{tabular}

Notes: Full Sample: all enrollees 27-64, from July 2007 to December 2009. Analysis Sample is first-time enrollees age 27-64 choosing individual coverage from Nov'09-Dec'09.

TABLE 2-CONDITIONAL LOGIT MODEL OF DEMAND

\begin{tabular}{|c|c|c|c|c|c|}
\hline & (1) & (2) & (3) & (4) & (5) \\
\hline \multirow[t]{2}{*}{ Monthly Premium (\$100's) } & $-0.766^{* * *}$ & $-0.311^{* * *}$ & $-2.760^{* * *}$ & $-2.197 * * *$ & $-2.462 * * *$ \\
\hline & $(0.0941)$ & $(0.112)$ & $(0.281)$ & $(0.189)$ & $(0.255)$ \\
\hline \multirow[t]{2}{*}{ Monthly Premium X Age (years) } & & & $0.0300^{* * *}$ & $0.0195^{* * *}$ & $0.0176^{* * *}$ \\
\hline & & & $(0.00386)$ & $(0.00301)$ & $(0.00335)$ \\
\hline \multirow[t]{2}{*}{ 1(Minimum Price Plan) } & $0.255^{* *}$ & $0.514^{* *}$ & $0.441^{* * *}$ & $0.419 * * *$ & 0.0424 \\
\hline & $(0.118)$ & $(0.221)$ & $(0.117)$ & $(0.116)$ & $(0.196)$ \\
\hline Fixed Effects & $\begin{array}{l}\text { Tier, } \\
\text { Insurer }\end{array}$ & Plan & Plan & $\begin{array}{l}\text { Tier x Age Trend, } \\
\text { Insurer }\end{array}$ & Plan x Age Trend \\
\hline $\mathrm{N}$ : Individuals & 1059 & 1059 & 1059 & 1059 & 1059 \\
\hline N: Plan Options x Individuals & 20,838 & 20,838 & 20,838 & 20,838 & 20,838 \\
\hline
\end{tabular}

Notes: Sample: Analysis Sample. Heteroskedasticity robust standard errors in parentheses. "Tier x Age Trend" includes a linear age trend in preference for each tier, and "Plan x Age Trend" includes a linear age trend in preference for each plan. *** Significant at the 1 percent level, ** Significant at the 5 percent level, * Significant at the 10 percent level.

TABLE 3-RANDOM COEFFICIENTS LOGIT MODEL OF DEMAND

\begin{tabular}{ll} 
TABLE 3-RANDOM COEFFICIENTS LOGIT MODEL OF DEMAND \\
\hline Monthly Premium (100s of Dollars), & $-2.969^{* * *}$ \\
...Mean of Distribution at Age 27 & $(0.286)$ \\
& $0.0348^{* * *}$ \\
...Shift of Mean: Coeff x (Age minus 27) & $(0.00507)$ \\
& $3.248^{* * *}$ \\
...Standard Deviation of Distribution & $(0.565)$ \\
& -0.212 \\
1(Minimum Price Plan) & $(0.206)$ \\
& Tier \& Carrier \\
Fixed Effects & 1059 \\
N Individuals & 20,838 \\
N Plan Options x Individuals &
\end{tabular}

Notes: Sample: Analysis Sample. Results of a random coefficients logit model, in which the premium coefficient is constrained to follow a lognormal distribution at age 27, and shifted by the "shift mean" coefficient for older ages. Mean and standard deviation are of the premium coefficient distribution, transformed from the log normal. Estimation via maximum simulated likelihood using Stata's mixlogit add in. Heteroskedasticity robust standard errors in parentheses. ${ }^{* * *}$ Significant at the 1 percent level, ** Significant at the 5 percent level, * Significant at the 10 percent level. 


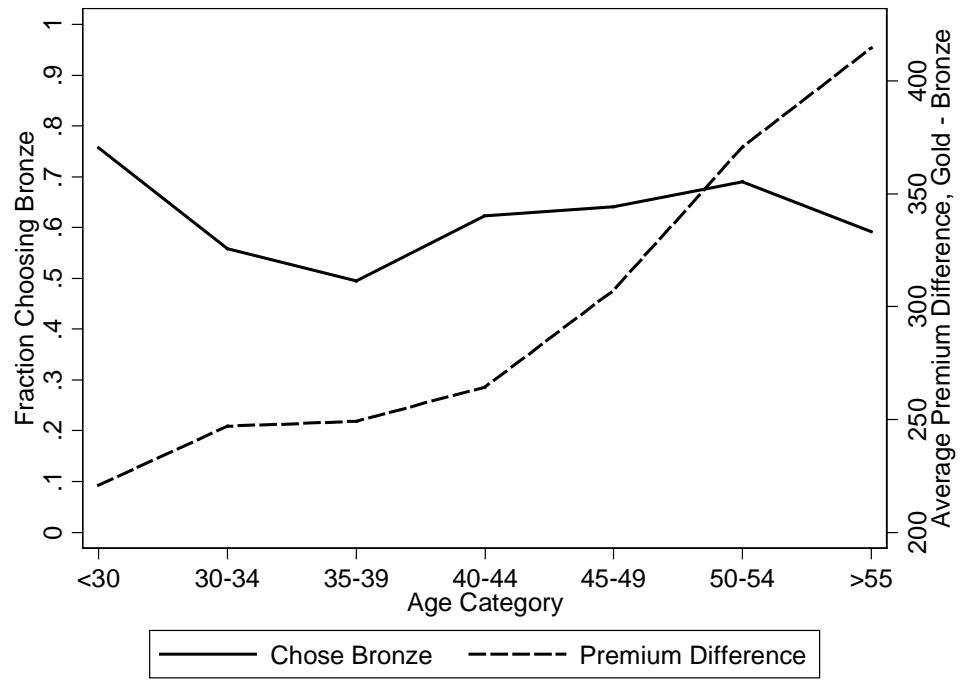

FIGURE 1 : AGE TRENDS IN CHOICE AND COST OF PLANS

Notes: Analysis Sample. Solid line shows the fraction of each age category choosing a bronze generosity plan. The dashed line shows the average monthly cost of a gold plan minus the average cost of a bronze plan, averaged over all enrollees in each age category. 